\title{
Norois
}

Environnement, aménagement, société

$203 \mid 2007 / 2$

Mobilité, hydrologie, loisir et tourisme, espace urbain

\section{Les étudiants étrangers : entre difficultés de la mesure et mesures restrictives. Une application à la Bretagne}

Foreign students: between difficulties of the measure and restrictive measures.

Application to Brittany

\section{Eugénie Terrier et Raymonde Séchet}

\section{OpenEdition}

Journals

Édition électronique

URL : https://journals.openedition.org/norois/1556

DOI : $10.4000 /$ norois. 1556

ISBN : 978-2-7535-1551-2

ISSN : 1760-8546

Éditeur

Presses universitaires de Rennes

Édition imprimée

Date de publication : 1 juin 2007

Pagination : 67-84

ISBN : 978-2-7535-0511-7

ISSN : 0029-182X

Référence électronique

Eugénie Terrier et Raymonde Séchet, «Les étudiants étrangers : entre difficultés de la mesure et mesures restrictives. Une application à la Bretagne », Norois [En ligne], 203 | 2007/2, mis en ligne le 01 juin 2009, consulté le 14 janvier 2022. URL : http://journals.openedition.org/norois/1556 ; DOI : https://doi.org/10.4000/norois. 1556 


\title{
LES ÉTUDIANTS ÉTRANGERS :
}

\section{ENTRE DIFFICULTÉS DE LA MESURE ET MESURES RESTRICTIVES UnE APPLiCATion À LA Bretagne}

\author{
Eugénie Terrier, Raymonde SÉchet \\ RESO - UMR ESO 6590 CNRS \\ (Université de Rennes 2), \\ Maison de la Recherche en Sciences sociales, \\ Place du Recteur-Henri-Le-Moal - 35043 RenNES cedex \\ engenie.terrier@uhb.fr, raymonde.sechet@uhb.fr
}

\section{RÉSUMÉ}

Les statistiques officielles sur la présence des étudiants étrangers dans l'enseignement supérieur français sont fondées sur le seul critère de la nationalité et font l'amalgame entre les étudiants issus des populations immigrées et les étudiants réellement mobiles. Or, face à la forte augmentation du nombre d'étudiants étrangers depuis 1998, et dans le contexte actuel de décentralisation des compétences, une bonne connaissance des données et de la condition sociale des étudiants est indispensable, tout particulièrement en Bretagne où les étudiants étrangers ont longtemps été peu nombreux. La population des étudiants étrangers est très hétérogène, ce qui rend plus difficile encore l'adaptation des structures d'accueil. L'évolution des effectifs et les inégalités de conditions des mobilités étudiantes sont fortement influencées par les politiques nationales, elles-mêmes soumises à des intérêts contradictoires.

MotS CLÉS : Analyse démographique - Bretagne - Mobilité étudiante - Outil statistique - Politiques d'accueil des étudiants étrangers.

\section{ABSTRACT}

\section{Foreign students: between difficulties of the measure and restrictive measu-} res. Application to Brittany

Official statistics about foreign students in French Higher Education are only based on nationality which means there is a confusion between students from families of migrants and genuine mobile students. Confronted to the important growth of the number of foreign students since 1998, and because of the current devolution of political competences, a good knowledge of data and of the social condition of students is essential, particularly in Brittany, where for many years few students used to come. Moreover, the population of foreign students is very heterogeneous, which makes the adaptation of host structures more difficult. The evolution of the number of foreign students and the inequalities between the conditions of student mobility are strongly influenced by national policies, which are themselves in the grips of contradictory interests.

KEY WORDS : Brittany - Demographic Analysis - Public Policies towards foreign students - Statistical Tool - Student Mobility. 
En 2004, 2,5 millions d'étudiants sont dans un pays autre que celui dont ils ont la nationalité, alors qu'ils n'étaient que 1,75 million cinq ans auparavant, ce qui représente une augmentation de 41 \% depuis 1999 (OCDE, 2003; UNESCO, 2003). Le début du XXI e siècle est donc marqué par une importante augmentation du nombre d'étudiants étrangers dans le monde. Avec $10 \%$ des effectifs mondiaux d'étudiants étrangers, la France est une des destinations préférentielles de ces migrations. Elle se trouve à la quatrième place après les États-Unis (23\%), le Royaume-Uni (12\%) et l'Allemagne (11\%). En 2004-2005, 255591 étudiants étrangers poursuivent leurs études dans l'enseignement supérieur français (DEP, 2005), soit environ 95000 de plus qu'en 1990 et $11,3 \%$ de l'ensemble des étudiants du pays. Cet afflux d'étudiants étrangers pose des questions politiques, économiques, sociales et culturelles et bouscule les discours sur la tradition d'accueil de la France.

Toutefois, la présence des étudiants étrangers sur le territoire national est très hétérogène. Dans le contexte actuel de décentralisation politique des compétences, l'échelon régional est un niveau d'observation pertinent. Les Régions jouent en effet un rôle croissant dans le pilotage de l'enseignement supérieur français, et ceci est particulièrement sensible en Bretagne ${ }^{1}$.

La Bretagne est-elle une région attractive pour les étudiants étrangers? Cette région est-elle touchée par la forte croissance des effectifs étrangers dans l'enseignement supérieur comme cela s'observe aux échelles nationale et mondiale? La présentation des effectifs étrangers inscrits dans l'enseignement supérieur en Bretagne est la première étape d'investigation d'un travail de thèse sur les mobilités et les expériences territoriales des étudiants étrangers en Bretagne ${ }^{2}$. Les données proviennent de différentes sources : la Direction de l'évaluation et de la prospective (DEP) du Ministère de l'Éducation nationale, le rectorat de Rennes, les universités de Bretagne et le CROUS (Centre Régional des Euvres Universitaires et Scolaires). Les étudiants en mobilité internationale, dont le séjour en France est lié à leurs études et à aucun autre motif (ni familial, ni de travail...), constituent un groupe difficilement repérable. La première partie de l'article est donc une réflexion sur les outils statistiques disponibles pour l'étude des mobilités étudiantes. Elle est suivie d'une présentation des données relatives aux étudiants en mobilité internationale inscrits dans l'enseignement supérieur en Bretagne. L'évolution de ces chiffres est ensuite, dans une dernière partie, replacée dans le contexte national, au niveau institutionnel où se décident les politiques d'accueil. Celles-ci sont actuellement en proie aux contradictions entre la volonté d'attirer des étudiants étrangers et l'adoption de mesures restrictives en matière de politiques migratoires.

\section{Les indicateurs statistiques de la mobilité étudiante}

Que cela soit dans la conversation courante ou dans les rapports officiels, l'expression « étudiant étranger » est communément utilisée pour désigner les étudiants en mobilité internationale. Certains chercheurs (Slama, 1999; Aubert et al., 1996) ont mis en avant le caractère ambivalent de cette expression ainsi que le flou statistique et les difficultés logistiques qu'une mauvaise catégorisation statistique pouvait engendrer. L'exemple des étudiants étrangers illustre bien le recul dont le chercheur doit faire preuve par rapport à l'arbitraire des catégories statistiques, celles-ci étant parfois extrêmement éloignées des réalités sociales.

\section{« ÉtUdiANT ÉTRANGER » : UNE CATÉgORIE STATISTIQUe CONFUSE}

La DEP (2005), service statistique du Ministère de l'Éducation nationale, définit l'étudiant étranger de la manière suivante : « Sont pris en compte dans les différentes enquêtes de recense-

1. Sur ce point, on pourra se référer à l'évaluation du programme Université 2000 et des volets « Enseignement supérieur et Recherche » des Contrats de Plan État-Région en Bretagne réalisée pour la Région Bretagne et la Préfecture de la Région Bretagne par F. Coutelier, J.-P. Domergue, D. Filâtre (Intermède - études et conseil), juillet 2002.

2. Cette thèse bénéficie d'un financement de la Région Bretagne. 
ment des étudiants inscrits en France ceux se déclarant de nationalité étrangère, ce qui inclut ceux ayant effectué leur scolarité du secondaire dans le système scolaire français. » Cette définition rassemble sous une seule appellation tous les étudiants n'ayant pas la nationalité française, soit deux groupes aux parcours de vie bien différents : les étudiants qui sont en mobilité internationale, c'est-à-dire ceux qui se rendent en France exclusivement pour leurs études, et les étudiants issus de l'immigration, n'ayant pas la nationalité française et dont les parents résident en France.

La catégorie statistique "étudiant étranger » qui recouvre des populations très différentes est donc peu satisfaisante. Il est fondamental de faire la distinction entre la population étudiante issue de l'immigration permanente et celle venant spécifiquement faire des études en France. Former un étranger, qui a vocation à rentrer dans son pays, et former un jeune qui, de quelque façon que ce soit, s’intégrera en France, sont deux réalités distinctes qui devraient avoir chacune leur indicateur statistique. Il s'agit de populations aux conditions de vie et au devenir très différents, l'une n'étant théoriquement que de passage ${ }^{3}$, et l'autre devant trouver sa place dans la société française, et même devenir française ${ }^{4}$. Ces deux populations d'étudiants sont pourtant appréhendées avec la même appellation générique par les pouvoirs publics. L'inadaptation des indicateurs statistiques à la réalité sociale fait régner une certaine confusion dans ce qui est dit autour de l'accueil des étudiants en mobilité internationale.

\section{UN FLOU STATISTIQUE À LA SOURCE DE LA MÉCONNAISSANCE DES FLUX}

L'OCDE (2003) fait le constat suivant en ce qui concerne les statistiques : «Dans la plupart des pays, on entend par étudiant étranger tout étudiant ne possédant pas la nationalité du pays pour lequel les données ont été recueillies. Cette définition est pragmatique et applicable mais elle peut donner lieu à des biais liés aux politiques nationales en matière de naturalisation des immigrants ou à l'incapacité de certains pays de déduire des effectifs d'étudiants étrangers ceux qui sont titulaires d'un permis de séjour permanent. En conséquence, les pays qui appliquent des politiques strictes en matière de naturalisation des immigrants et qui sont dans l'incapacité d'identifier les étudiants étrangers non-résidents surestiment l'importance des effectifs d'étudiants étrangers par rapport aux pays qui appliquent des dispositions moins restrictives en matière de naturalisation. »

L'Allemagne figure parmi les destinations favorites des étudiants étrangers mais les étudiants non-résidents ne représentent que les deux tiers des étudiants étrangers inscrits dans les établissements de l'enseignement supérieur du pays. Ce phénomène s'explique par la proportion importante de « résidents étrangers », correspondant principalement à des enfants de travailleurs immigrés. Dans le cadre de cet indicateur, ces personnes sont considérées comme "étrangères » même si elles ont grandi en Allemagne et qu'elles y résident en permanence. Pour la France, plusieurs rapports et recherches (Jallade et Gordon, 1996; Borgogno et Vollenweider, 1998; Teissier et al., 2004) ont constaté une même surestimation du nombre d'étudiants étrangers. Cette confusion est évitée au Royaume-Uni, où la préoccupation n’est pas la nationalité mais le pays de résidence permanente et où les étudiants sont considérés comme non-ressortissants s'ils ont leur résidence principale dans un autre pays alors que pour les autres États membres de l’Union européenne, les étudiants non-ressortissants sont ceux qui possèdent un passeport d'un autre pays.

3. La possibilité du non-retour des étudiants étrangers s'explique souvent par l'inadéquation entre le marché du travail dans le pays d'origine et le niveau de qualification de l'étudiant formé en France. Selon B. Laffort (2005), seulement $50 \%$ des étudiants marocains rentreraient au pays après leurs études.

4. Selon la définition adoptée par le Haut Conseil à l'Intégration, un immigré est une personne née étrangère à l'étranger et résidant en France. Les personnes nées françaises à l'étranger et vivant en France ne sont donc pas comptabilisées. A l'inverse, certains immigrés ont pu devenir français, les autres restant étrangers. Les populations étrangère et immigrée ne se confondent pas totalement : un immigré n'est pas nécessairement étranger et réciproquement certains étrangers sont nés en France (essentiellement des mineurs). La qualité d'immigré est permanente : un individu continue à appartenir à la population immigrée même s'il devient français par acquisition. C'est le pays de naissance, et non la nationalité à la naissance, qui définit l'origine géographique d'un immigré. Un étranger est une personne qui n'est pas ressortissant de la nation où il vit. 
Pourquoi un tel attachement statistique au critère de la nationalité en France alors que celui-ci ne permet pas une appréhension adéquate de la réalité? Selon F. Aubert et al. (1996), « ce que certains considèrent comme une lacune, d'autres comme une garantie fondamentale des libertés, a notamment pour origine notre conception "non ethnique, non raciale, non religieuse" de la nationalité qui s'appuie sur un critère juridique abstrait. C'est la traduction, dans notre appareil statistique national, des fondements de la République et de la citoyenneté ${ }^{5}$ ».

\section{LES VOIES DE LA CONNAISSANCE ET LEURS LIMITES}

Relevant que «sur le plan quantitatif et statistique, la mobilité des étudiants est mal connue et difficile à appréhender », les ministres de l'Éducation réunis en conseil le 9 novembre 1993, « exprimèrent le besoin de disposer d'une information adéquate sur les flux d'étudiants mobiles au sein de l'Union Européenne ». Pour ce faire, ils confièrent à J. Gordon et J.-P. Jallade, de l'Institut européen d'éducation et de politique sociale, la mission de procéder à une enquête à ce sujet. Dans leur rapport remis en 1996, ceux-ci ont pris comme parti de réaliser une enquête dont la caractéristique « serait de distinguer les étudiants «étrangers résidents » des étudiants " étrangers mobiles ", seuls les seconds étant retenus pour comptabiliser les flux de mobilité étudiante au sein de l'Union Européenne » (Jallade et Gordon, 1996). Pour cela, ils ont fait appel « aux bureaux nationaux des statistiques » qui « collectent, au niveau national, les statistiques officielles provenant des universités ». Pour la France, il s'agit de la DEP, à laquelle il a été demandé de soustraire les étudiants « étrangers résidents » du total des étudiants étrangers de manière à obtenir les étudiants «étrangers mobiles ». Comment la DEP a-t-elle donc fait pour répondre à cette demande, alors que la distinction n'est pas établie dans les statistiques qu'elle publie? La note technique du rapport indique que, pour la France, « les étudiants étrangers sont ceux qui n'ont pas la nationalité française ", ce qui correspond à la définition de l'étudiant étranger traditionnellement retenue par la DEP. Et « les étudiants mobiles ont un diplôme d'études secondaires étranger équivalent au baccalauréat ». Les étudiants étrangers « mobiles » sont donc distingués des résidents au moyen des statistiques fournies par les universités sur les diplômes d'études secondaires étrangers. La DEP peut donc isoler statistiquement cette population d'étudiants «mobiles ». La technique utilisée pour cette évaluation n'est toutefois pas entièrement fiable, puisqu'un certain nombre d'étudiants étrangers «mobiles » peuvent avoir un diplôme français : par exemple ceux qui sont venus au cours de leurs études secondaires en France ou ceux qui sont arrivés avec un baccalauréat obtenu dans un lycée français de l'étranger. Cette méthode sous-évalue donc probablement la population réelle d'étudiants «mobiles ». Elle permet cependant de se faire une idée de son importance dans l'ensemble de la population d'étudiants de nationalité étrangère.

Depuis les recherches évoquées précédemment, certains rapports officiels concernant les étudiants étrangers incluent cette distinction entre les étudiants mobiles et les étudiants issus de l'immigration. Dans le dernier rapport publié à ce jour par la DEP, intitulé Les étudiants étrangers en France (Teissier et al., 2004), certaines parties de l'analyse tiennent compte de la différence entre étudiants immigrés et étudiants expatriés. La distinction est faite à partir de la variable « lieu d'obtention du baccalauréat »; c'est pourquoi ce rapport nomme les étudiants étrangers en mobilité internationale « les étudiants étrangers non-bacheliers ». Cette distinction n'est toutefois pas réalisable pour des analyses à un niveau fin et portant sur la totalité des effectifs de l'enseignement supérieur.

Ainsi, lors de l'évaluation des effectifs d'étudiants étrangers dans l'ensemble de l'enseignement supérieur en Bretagne (c'est-à-dire universités et hors universités), il n'a pas été possible de faire la distinction entre les étudiants étrangers mobiles et ceux issus de l'immigration. En effet, les statistiques fournies au rectorat par les établissements hors université sont très peu détaillées et

5. Ce débat renvoie aussi à la question de la prise en compte du critère de «l'ethnicité » dans les statistiques concernant les populations immigrées en France. 
se limitent souvent à la distinction « français/étrangers ». En ce qui concerne les universités et établissements assimilés où $87 \%$ des étudiants étrangers sont inscrits en 2005, il a été possible de remédier à la faiblesse de la catégorie statistique "étudiants étrangers » grâce à une analyse fine des fichiers détaillés fournis par la DEP et les universités elles-mêmes. Ces chiffres ont permis d'estimer le nombre d'étudiants étrangers véritablement mobiles (les étudiants étrangers expatriés - EEE) au sein des universités de Bretagne. Les effectifs « réels » d'étudiants étrangers en Bretagne ont été calculés grâce à la variable « série du baccalauréat » du $\mathrm{SISE}^{6}$; celle-ci nous renseigne sur le lieu d'obtention du diplôme secondaire qui donne l'accès à l'enseignement supérieur. Les étudiants étrangers issus de l'immigration indiquent la série de leur baccalauréat passé en France (S, L, ES, STI, STT, etc.) alors que les étudiants étrangers réellement mobiles précisent que leur diplôme est étranger. Ce travail a permis de repérer les étudiants mobiles, ceux qui ont réalisé leur scolarité à l'étranger et qui viennent en France spécifiquement pour les études. La surestimation du nombre d'étudiants étrangers en Bretagne a ainsi pu être corrigée. Il s'agit cependant d'une approximation dans la mesure où il est possible de passer le baccalauréat à l'étranger. Nous n'avons pas retenu la variable «lieu de résidence des parents » en raison de l'ambiguïté dans le dossier d'inscription de l'étudiant sur la notion d'« adresse permanente » qui peut aussi être interprétée comme l'adresse où l'étudiant peut être joint pendant les vacances scolaires.

\section{La Bretagne : une région attractive pour les étudiants en mobilité internationale?}

Malgré un nombre relativement faible d'étudiants étrangers par rapport à d'autres régions, la Bretagne représente un cas d'étude très intéressant. La diversité de son offre universitaire (Rennes 2, Rennes 1, Brest, Bretagne-Sud) permet d'aborder la question de l'effet de site dans les orientations des étudiants étrangers. De plus, parce qu'elle accueille de nombreux étudiants ${ }^{7}$ mais très peu d'immigrés, la région Bretagne est un cas intéressant dans le contexte actuel de très forte croissance des effectifs d'étudiants étrangers en France et dans la région.

\section{DES ÉTUDIANTS ÉTRANGERS PEU NOMBREUX ET INÉGALEMENT RÉPARTIS}

En 2004-2005, environ 6700 étudiants de nationalité étrangère étaient inscrits dans l'enseignement supérieur en Bretagne, dont $87 \%$ dans l'une des quatre universités contre $62 \%$ des étudiants français (tableau 1). Les autres étudiants étrangers sont majoritairement inscrits dans les écoles d'ingénieurs et les écoles de commerce; ils sont très peu nombreux dans les sections de techniciens supérieurs des lycées. Dans le cadre de cette étude sur les étudiants étrangers en Bretagne, nous ne pouvons pas retenir cet effectif de 6700 qui surestime la population qui nous intéresse, c'est-à-dire les étudiants étrangers réellement mobiles. La relecture des statistiques, avec la prise en compte de la variable «lieu d'obtention du baccalauréat », montre qu'en 2004-2005, 71,2 \% des étudiants de nationalité étrangère inscrits dans les universités bretonnes, soit 4173 individus, étaient des étudiants étrangers expatriés.

En 2004-2005, les étudiants en mobilité internationale représentaient ainsi 6,1\% de l'ensemble des étudiants inscrits dans les universités bretonnes. Cette part d'étudiants étrangers expatriés est presque deux fois moins élevée que celle calculée à l'échelle de la France (10,5\%). Si l'on compare ces chiffres avec les effectifs d'étudiants étrangers dans les autres régions françaises, il s'avère que la Bretagne reçoit relativement peu d'étudiants étrangers (fig. 1). La France accueillait environ 150000 étudiants étrangers en mobilité en 2004-2005. La répartition de ces étudiants étrangers mobiles sur le territoire national est plus concentrée que celle des étudiants français :

6. Le système d'information sur le suivi de l'étudiant (SISE) a remplacé depuis 1994, le système « effectifs universitaires (EFU)».

7. Avec 109925 étudiants inscrits en 2004-2005, la Bretagne se situe à la sixième place sur 22 régions. 


\begin{tabular}{|l|l|l|l|}
\hline & $\begin{array}{l}\text { Ensemble de l'ensei- } \\
\text { gnement supérieur } \\
\text { de Bretagne }\end{array}$ & $\begin{array}{l}\text { Universités et assi- } \\
\text { milés }\end{array}$ & $\begin{array}{l}\text { Part d'étudiants à } \\
\text { l'Université }\end{array}$ \\
\hline Total étudiants (français et étrangers) & 109925 & 68227 & $62,0 \%$ \\
\hline Étudiants de nationalité étrangère (en nombre) & 6685 & 5865 & $87 \%$ \\
\hline Étudiants de nationalité étrangère (en \%) & 6,1 & 8,6 & \\
\hline Étudiants étrangers expatriés (EEE) en nombre & & 4173 & \\
\hline Étudiants étrangers expatriés (EEE) en \% & & 6,1 & 71,2 \\
\hline Part des EEE/Total des étudiants étrangers (en \%) & & & \\
\hline
\end{tabular}

Tableau 1 : Les effectifs d'étudiants étrangers dans l'enseignement supérieur en Bretagne en 2004 Number of foreign students in Higher Education in Brittany in 2004

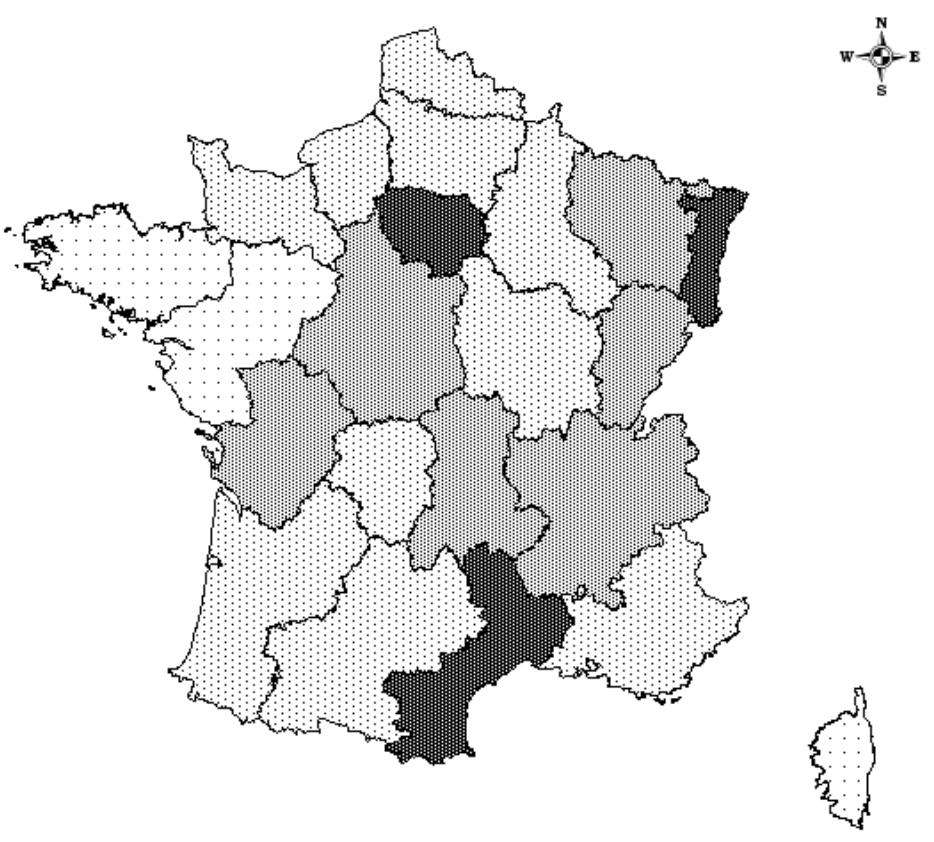

\section{Part des étudiants étrangers mobiles}

(en pourcentage de l'ensemble des étudiants de la région)

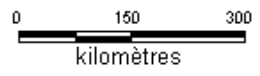

kilomètres

Figure 1 : Proportion d'étudiants étrangers expatriés par région française (Universités, 2005) Internationally mobile students ratio by french region (Universities, 2005) 
neuf académies accueillent les deux tiers des étudiants étrangers. Seulement 2,8\% de l'ensemble des étudiants étrangers expatriés sont inscrits en Bretagne, alors que les trois académies d'Ile-deFrance accueillent à elles seules plus d'un étudiant étranger sur trois (34\% du total des EEE).

La répartition spatiale des étudiants en mobilité internationale est à mettre en parallèle avec la carte de la présence d'étrangers et d'immigrés en France. En effet, l'Île-de-France et les régions du Sud-Est, qui ont une forte tradition d'accueil de populations immigrées et étrangères, sont également celles où les étudiants étrangers expatriés sont les plus nombreux. L'étude de V. Borgogno et L. Vollenweider (1998) qui s'est concentrée sur les étudiants étrangers maghrébins, nous indique qu'il existe des liens entre les étudiants étrangers « expatriés » et les communautés maghrébines issues de l'immigration. Dans l'enquête qu'ils ont menée, $74 \%$ des interrogés déclarent avoir eu de la famille en France avant leur arrivée. La présence de personnes de la même famille ou de la même communauté peut donc influer sur le choix de la ville d'étude. Cette proximité permet à l'étudiant étranger de disposer d'un réseau relationnel grâce aux compatriotes déjà établis. Ces derniers peuvent jouer un rôle fondamental dans la détermination des conditions d'existence de l'étudiant étranger en lui apportant une aide multiforme (matérielle, économique, dans les démarches administratives, pour trouver un job...). L'aide que ces étudiants sont susceptibles de recevoir des réseaux familiaux et sociaux au sein des communautés immigrées en France facilite le séjour de l'étudiant étranger en venant alléger de manière non négligeable d'éventuels problèmes d'adaptation sociale, culturelle et linguistique. Ainsi, lorsque nous savons que la Bretagne accueille très peu d’immigrés (en 1999, les immigrés ne représentaient que 1,6 \% de la population bretonne contre 7,4\% pour l'ensemble de la population française), nous pouvons faire l'hypothèse qu'il existe un rapport entre la faible tradition d'accueil d'étudiants étrangers en Bretagne et cette faible proportion d'immigrés.

À l'instar de l'ensemble des étudiants, les étudiants étrangers expatriés sont très inégalement répartis sur le territoire breton. Rennes et Brest regroupent à elles seules $91 \%$ des étudiants étrangers expatriés présents en Bretagne (fig. 2). La concentration des étudiants étrangers expatriés est donc encore plus forte que celle de l'ensemble des étudiants puisque les établissements de Rennes et de Brest accueillent $88 \%$ de l'ensemble des étudiants de Bretagne. Notons que Rennes, en tant que capitale régionale et universitaire, se démarque nettement en accueillant 10 étudiants étrangers sur 15. Cela fait un total de 2857 étudiants étrangers expatriés à Rennes en 2005, contre 1138 étudiants étrangers expatriés à Brest et 236 à Lorient. Le solde restant se répartit entre Vannes, Quimper, Lannion, Saint-Malo, Saint-Brieuc et Morlaix.

\section{DES EFFECTIFS EN FORTE CROISSANCE}

Les données fournies par le Rectorat et par la DEP permettent une étude diachronique depuis 1964 sans toutefois pouvoir isoler les étudiants étrangers expatriés. En effet, les chiffres détaillés permettant la distinction entre les étudiants étrangers expatriés et les étudiants étrangers immigrés n'existent que depuis 1996, date de la création du logiciel SISE. Les chiffres disponibles sur les étudiants de nationalité étrangère permettent cependant une estimation fiable de la croissance des effectifs. Le nombre d'étudiants étrangers en Bretagne augmente très fortement entre 1964 et 2005 (fig. 3) : en quarante années, celui-ci a été multiplié par 15. Alors qu'en 1964 il n’y avait que 389 étudiants étrangers dans les universités de l'académie de Rennes, ceux-ci sont aujourd'hui 5878. La courbe d'évolution des effectifs d'étudiants étrangers en Bretagne montre que la croissance s'est faite selon trois périodes.

De 1964 à 1985, l'augmentation du nombre d'étudiants étrangers a été relativement forte. Les effectifs ont été multipliés par 6 en 20 ans et dépassent les 2500 individus en 1985. Cette croissance des effectifs correspond à l'arrivée massive des étudiants en provenance des anciennes colonies françaises. 


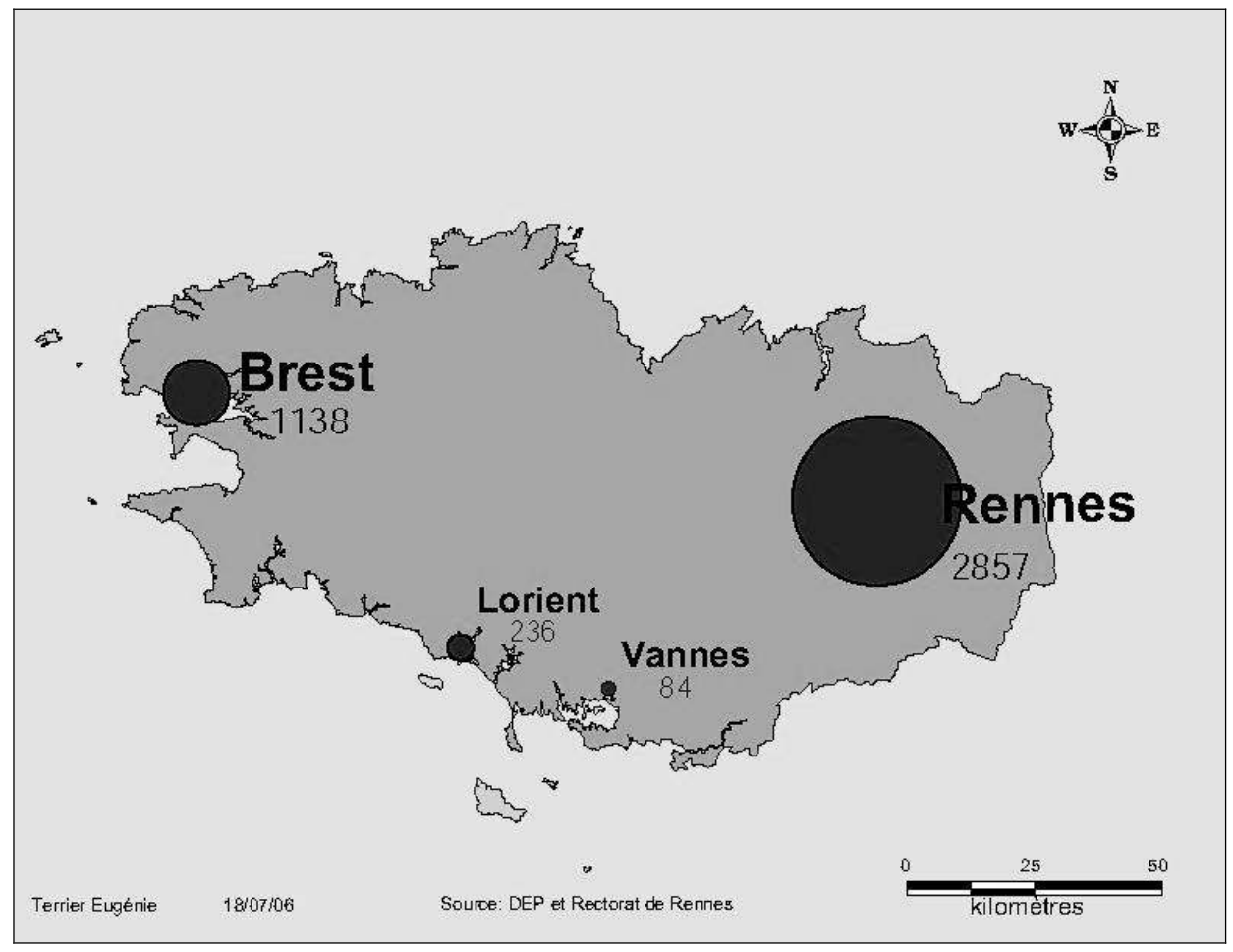

Figure 2 : Nombre d'étudiants étrangers expatriés par ville bretonne (Universités, 2005) Number of internationally mobile students in cities in Brittany (Universities, 2005)

De 1985 à 1997, le nombre d'étudiants étrangers a diminué, passant de 2503 à 2370 individus, alors que dans le même temps les effectifs totaux progressaient fortement. Cette période de stagnation du nombre d'étudiants étrangers en Bretagne - et de réduction significative de leur part dans le total des effectifs - est constituée d'une succession d'années de régression et d'années de légère reprise des effectifs. Les mesures restrictives en matière de conditions d'accueil et de séjour des étudiants étrangers, particulièrement envers ceux soupçonnés de comporter un risque migratoire (ceux issus des pays du Sud) expliquent ces années de régression des effectifs. La légère reprise de la croissance entre 1988 et 1993 correspond à la progression du nombre d'étudiants issus de l’immigration ainsi qu'à l'européanisation des effectifs.

Enfin, la période 1997-2005 a été marquée par une très forte reprise de la croissance des effectifs. Le nombre d'étudiants étrangers en Bretagne a été multiplié par deux en seulement huit ans, passant de 2370 à 5878 individus. Ce retournement de situation est dû pour l'essentiel à l'assouplissement des conditions d'accueil des étudiants étrangers sur le sol français avec, par exemple, la facilitation de l'obtention du visa décidée en 1998 par le gouvernement Jospin ${ }^{8}$. Notons cependant un ralentissement de cette croissance à partir de 2003.

L'isolement des seuls étudiants étrangers expatriés inscrits à l'université permet de mieux voir cette forte croissance des effectifs. Ils étaient 1092 en 1996; ils sont aujourd'hui 4380 (tableau n² 2). Les effectifs ont donc été multipliés par quatre en neuf ans seulement. La part des étudiants étrangers expatriés a connu une croissance considérable. Alors que ceux-ci étaient minoritaires en 1996 par rapport aux étudiants étrangers issus de l'immigration, ils sont aujourd'hui largement majoritaires : en 2005, plus de sept étudiants étrangers sur dix étaient des étudiants étrangers expatriés contre seule- 
ment quatre en 1996. Si l'on compare le taux de croissance des étudiants étrangers expatriés de Bretagne avec celui de la France entre 1998 et 2004, il s'avère que celui-ci dépasse le taux de croissance moyen à l'échelle nationale. Les effectifs d'étudiants étrangers expatriés en Bretagne ont connu une croissance de $+186 \%$ entre 1998 et 2004 contre $+120 \%$ pour la France. Malgré une faible proportion d'étudiants étrangers, la Bretagne fait partie des académies qui connaissent les plus fortes croissances du nombre d'étudiants étrangers expatriés. À l'inverse, dans les académies du Sud de la France, le nombre d'étudiants étrangers expatriés est important, mais les évolutions sont plus modérées.

Malgré la faible proportion d'étudiants étrangers qu'elle a connue jusqu'à aujourd'hui, la Bretagne possède un potentiel qu'il lui reste à exploiter. La croissance des effectifs oblige les pouvoirs publics de la région et des villes bretonnes, ainsi que les universités, à s'adapter à cette nouvelle situation, et impose de chercher à mieux connaître cette catégorie d'étudiants que sont les étudiants étrangers mobiles.

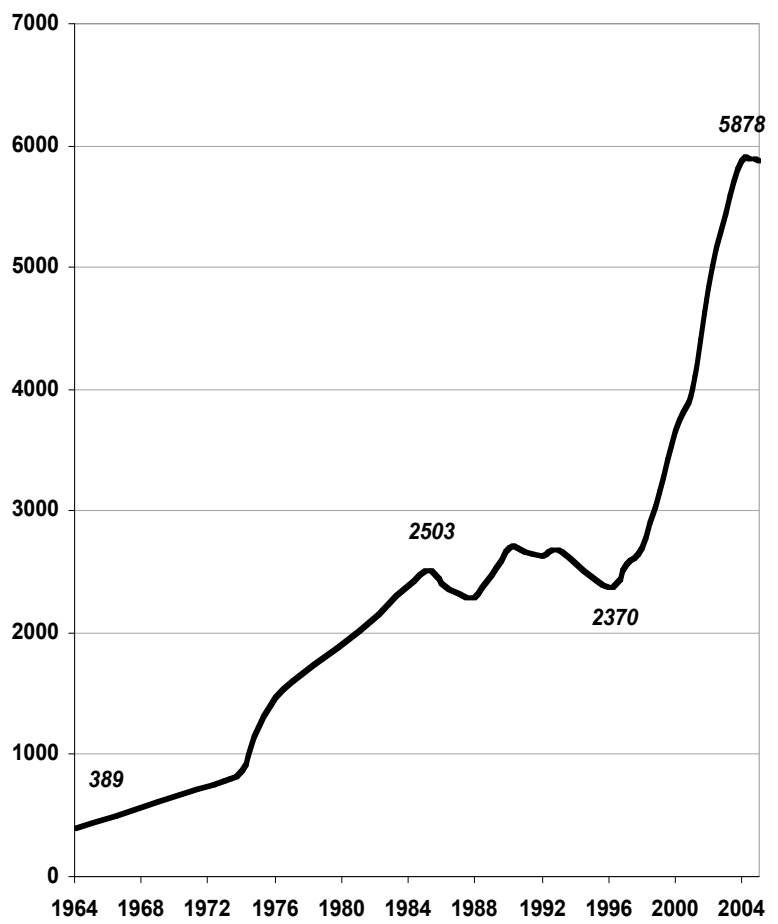

Figure 3 : Évolution des effectifs d'étudiants étrangers dans les universités bretonnes (1964-2005) (sources : DEP et Rectorat)

Evolution in the number of foreign students in University in Brittany (1964-2005)

\begin{tabular}{|l|l|l|l|l|l|l|l|l|l|l|}
\hline & 1996 & 1997 & 1998 & 1999 & 2000 & 2001 & 2002 & 2003 & 2004 & 2005 \\
\hline étudiants (total) & 69716 & 68473 & 67768 & 67320 & 67465 & 65870 & 67152 & 69185 & 68227 & 69780 \\
\hline étudiants étrangers (EEE+EEI) & 2370 & 2558 & 2692 & 3158 & 3651 & 4016 & 4830 & 5451 & 5865 & 5878 \\
\hline total EEE & 1092 & 1238 & 1457 & 1755 & 2087 & 2590 & 3224 & 3854 & 4173 & 4380 \\
\hline EEE/total étudiants étrangers & 46,1 & 48,4 & 54,1 & 55,6 & 57,2 & 64,5 & 66,7 & 70,7 & 71,2 & 74,5 \\
\hline EEE/total étudiants & 1,6 & 1,8 & 2,1 & 2,6 & 3,1 & 3,9 & 4,8 & 5,6 & 6,1 & 6,3 \\
\hline
\end{tabular}

Tableau 2 : Évolution des effectifs d'étudiants étrangers expatriés dans les universités de Bretagne (1996-2005) (Source : DEP)

Évolution in the number of internationally mobile students in Universities in Brittany (1996-2005)

\section{LA DIVERSITÉ DES ÉTUDIANTS ÉTRANGERS MOBILES}

Quatre étudiants étrangers (EEE) sur dix présents en Bretagne sont originaires du continent africain (fig. 4). L'Europe est le second continent le mieux représenté : environ trois étudiants étrangers sur dix sont européens. En moyenne, sur ces trois étudiants européens, deux sont originaires d'un pays de l'Union Européenne. Les étudiants étrangers mobiles originaires du continent asia- 


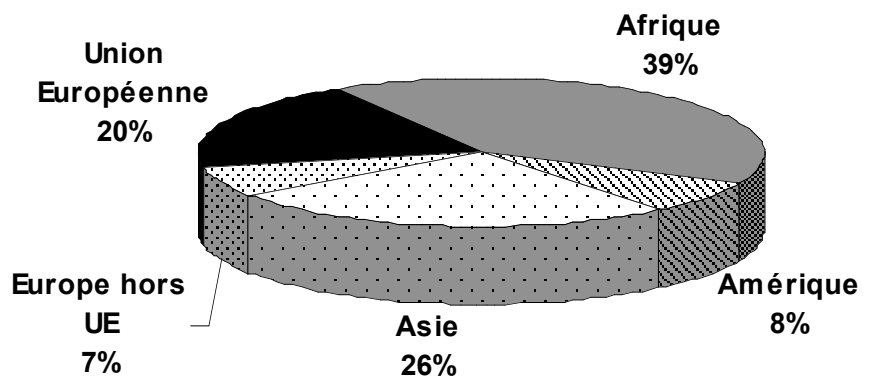

Figure 4 : Continent d'origine des étudiants étrangers expatriés en Bretagne (Universités, 2005) (Source : DEP)

Continent of origin of internationally mobile students in Brittany (Universities, 2005)

tique sont aussi nombreux que les étudiants étrangers européens (26\%). L’Afrique francophone, la Chine et l'Europe de l'Ouest sont donc les parties du monde les mieux représentées parmi les étudiants étrangers en Bretagne. Du point de vue de la nationalité, les étudiants originaires du Maroc sont les plus présents : en effet, deux étudiants étrangers sur dix sont marocains ${ }^{9}$.

Comme à l'échelle nationale, la très forte croissance depuis 1997 du nombre d'étudiants étrangers expatriés présents en Bretagne est principalement due à l'arrivée importante d'étudiants étrangers d'Asie et d'Afrique (fig. 5). Les étudiants étrangers asiatiques sont de plus en plus nombreux en Bretagne, particulièrement depuis l'année 2000. Ils n'étaient que 145 en 1996, ils sont aujourd'hui 1 136. Cela représente une croissance de $683 \%$, soit le plus fort taux de variation de tous les continents. Les Asiatiques ont même dépassé les effectifs d'étudiants en provenance de l'Union Européenne en 2001-2002. Ces étudiants asiatiques qui arrivent très nombreux en Bretagne sont pour la plupart originaires de Chine. Les effectifs d'étudiants étrangers africains (EEE) présents en Bretagne ont été multipliés par six en neuf années, passant de 306 individus à 1753 , soit une croissance de $473 \%$.

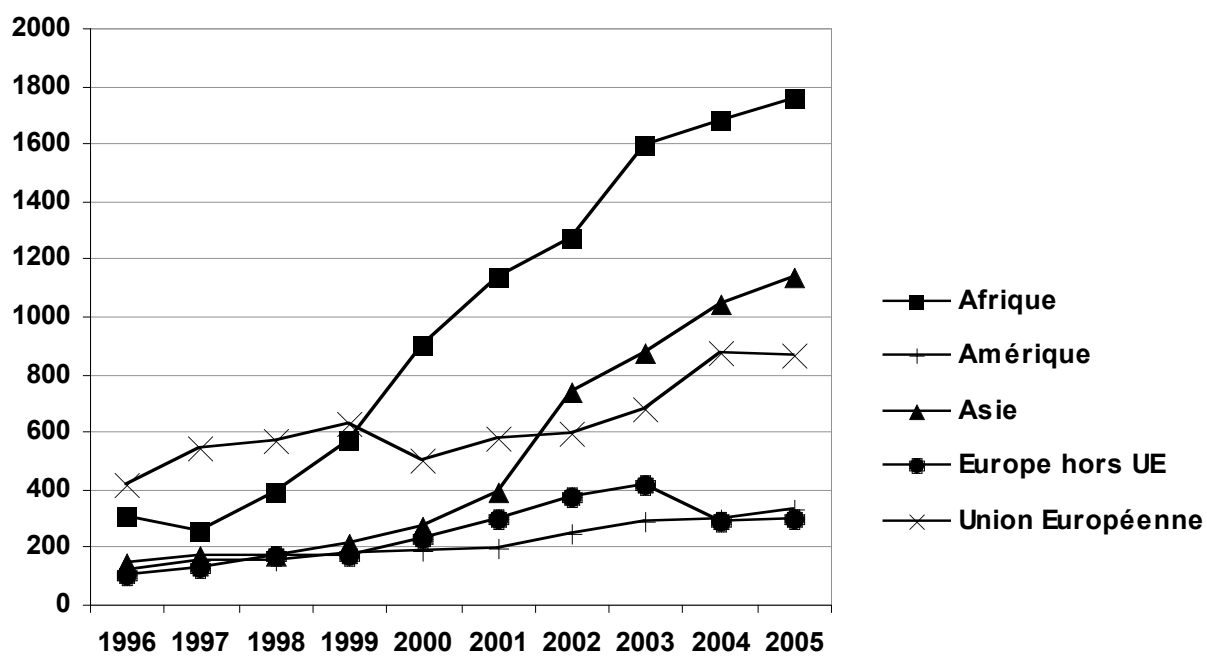

Figure 5 : Évolution du nombre d'étudiants étrangers expatriés en Bretagne selon leur continent d'origine (Universités, 1996-2005) (Source : DEP)

Evolution in the number of internationally mobile students in Brittany by continent of origin (Universities, 2005)

9. Les données par nationalité sont moins complètes que les données par continent d'origine. La distinction entre étudiants étrangers expatriés et étudiants étrangers immigrés n’était donc pas réalisable. 
La parité des sexes observée pour l'ensemble des étudiants étrangers (49\% d'étudiantes pour $51 \%$ d'étudiants) cache de fortes disparités selon les nationalités (fig. 6). Seuls les Asiatiques conservent une certaine égalité de représentation entre les hommes et les femmes. En revanche, les étudiants étrangers issus des pays européens (hors UE) sont à $74 \%$ des femmes. Ces dernières sont par contre sous-représentées chez les étudiants africains. Sur dix étudiants étrangers originaires du continent africain, il n'y a que trois femmes. Cette inégalité trouve en grande partie son origine dans les écarts d'accès à l'enseignement supérieur dans le pays d'origine selon le genre. Par exemple, seulement $21 \%$ des étudiants mauritaniens sont des femmes. Dans ces sociétés où la domination masculine prévaut, il est d'usage que les hommes soient prioritaires face aux études.

\section{Hommes $\square$ Femmes}

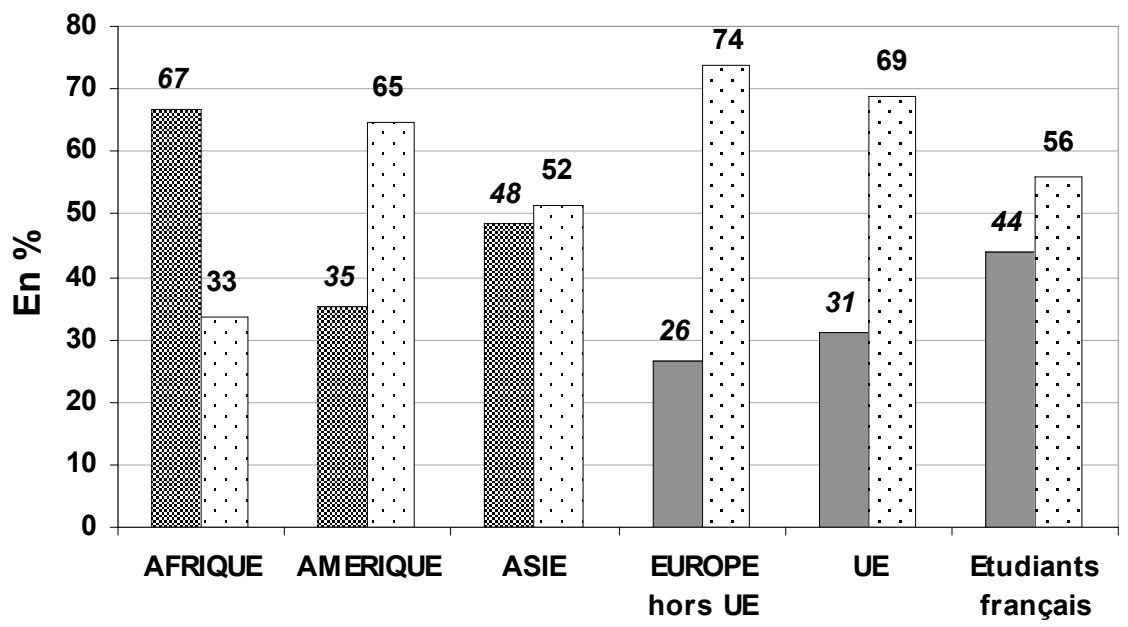

Figure 6 : Les étudiants étrangers mobiles en Bretagne par genre et par continent d'origine (Universités, 2005)

Number of internationally mobile students in Brittany by gender and by continent of origin (Universities, 2005)

À l’image des étudiants français, les étudiants étrangers s'inscrivent souvent en Lettres et en Sciences Humaines (36 \%). En revanche, les étudiants étrangers sont plus nombreux à s'inscrire en sciences que les étudiants français $(31 \%$ contre $24 \%$ ) et sont deux fois plus présents en sciences économiques. Notons enfin que les IUT ne regroupent que $2 \%$ des étudiants étrangers contre $10 \%$ des étudiants français. Finalement, la répartition de l'ensemble des étudiants étrangers par groupes de disciplines est relativement proche de celle des étudiants français. En revanche, si l'on s'intéresse à chaque nationalité, il s'avère que les disciplines de prédilection diffèrent d'un continent d'origine à l'autre (fig. 7). Selon A. Coulon et S. Paivandi « La quête de connaissance générale est beaucoup plus fréquemment observée dans le cas des étudiants issus des pays développés » (Coulon et Paivandi, 2003). Sept étudiants américains ${ }^{10}$ sur dix et plus de six étudiants de l'UE sur dix s'inscrivent en lettres et en sciences humaines, contre seulement un étudiant africain sur

10. Les statistiques disponibles pour l'ensemble de la région ne fournissent pas la nationalité détaillée des étudiants étrangers. Il faudra alors comparer pendant le travail de thèse les étudiants originaires d'Amérique du Nord avec ceux d’Amérique du Sud. 


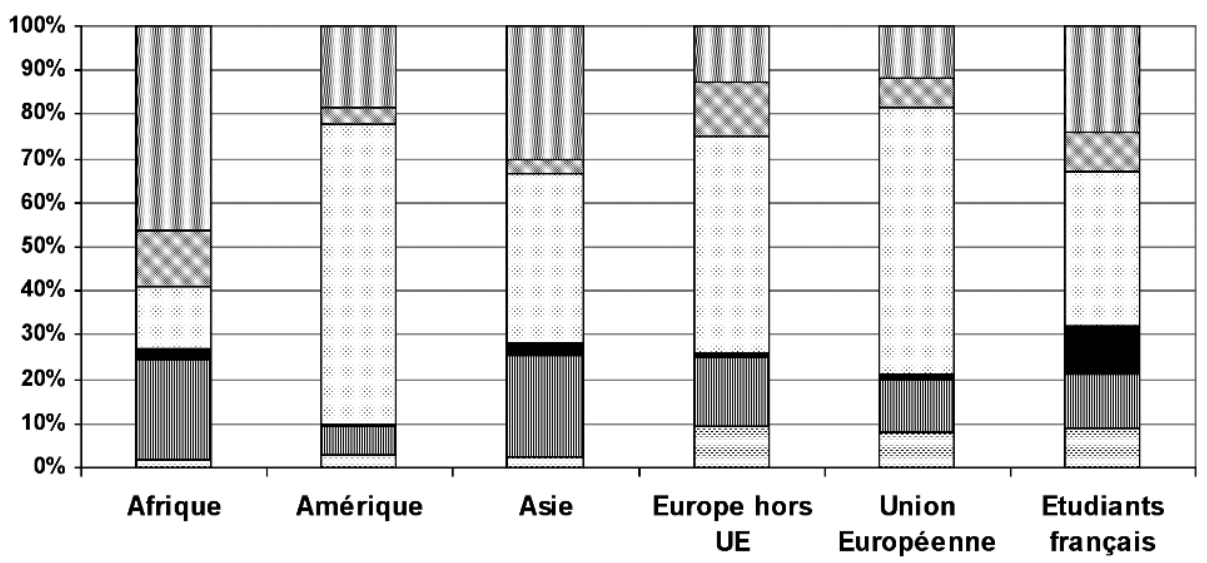

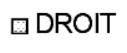

口IUT 口MEDECINE, ODONTOLOGIE

\section{m ECONOMIE AES \\ 口LETIRES, SCIENCES HUMAINES \\ $\square$ SCIENCES, STAPS}

Figure 7 : Répartition des étudiants étrangers mobiles selon le continent d'origine et la discipline (Universités bretonnes, 2005)

Distribution of the internationally mobile students by continent of origin and by discipline (Universities, 2005)

dix. Les connaissances scientifiques et les compétences techniques font partie de la demande des pays du Sud : $45 \%$ des étudiants africains sont inscrits en sciences contre seulement $10 \%$ des étudiants européens. Ceci s'explique en partie par les insuffisances en formations et en moyens des universités africaines, d'autant plus que les filières scientifiques nécessitent plus de moyens financiers que les filières littéraires (matériel d'expérimentation...). De plus, nous pouvons supposer que les motivations pour ces disciplines relèvent davantage d'un choix utilitaire dans une optique de développement économique ou technologique auquel ces étudiants africains pourront contribuer lorsqu'ils seront de retour dans leur pays d'origine.

Selon les données fournies par l'Université Rennes 2, environ deux étudiants étrangers sur 10 sont encadrés par un programme d'échange. Les statistiques concernant les étudiants étrangers de l'Université de Rennes 1 viennent confirmer cette tendance : en 2003, $23 \%$ des étudiants étrangers inscrits dans cet établissement sont venus dans le cadre d'un programme d'échange. En 2004, sur les 261 étudiants étrangers encadrés par un programme d'échange, 190 sont des étudiants Erasmus, soit $73 \%$ de l'ensemble des étudiants encadrés. Les autres étudiants étrangers sont venus dans le cadre d'accords bilatéraux passés entre l'université Rennes 2 et des universités étrangères. À Rennes 1, les étudiants étrangers encadrés par des programmes d'échanges sont en grande majorité des Européens et des Chinois.

En dehors de l'encadrement par un programme, une autre solution s'offre aux étudiants étrangers pour faciliter leur mobilité : les bourses. Pour l'instant, seule l'université Rennes 1 a fourni des indications statistiques sur la proportion de boursiers du gouvernement français et de gouvernements étrangers parmi les étudiants étrangers. En 2003, seulement $6 \%$ des étudiants étrangers mobiles inscrits à Rennes 1 sont boursiers. Parmi eux, environ $50 \%$ sont boursiers du gouvernement français et l'autre moitié d'un gouvernement étranger. Plus de la moitié des étudiants étrangers boursiers sont des Africains. Cependant, parmi les Africains, seulement 6,7 \% sont boursiers. Sachant que ces derniers sont rarement concernés par les programmes d'échanges, nous pouvons 
supposer que les étudiants africains qui viennent en Bretagne le font en très grande majorité à titre individuel. De plus, les chiffres montrent que la proportion de boursiers parmi les étudiants étrangers diminue fortement au fil des années. En effet, entre 1998 et 2003, la part de boursiers a été divisée par trois à Rennes 1 . Dans cet établissement, le nombre de bourses accordées par le gouvernement français et par les gouvernements étrangers n'a donc pas suivi l'évolution des effectifs d'étudiants étrangers.

Les proportions d'étudiants encadrés et d'étudiants boursiers connaissent une forte baisse depuis 1998, ce qui signifie une augmentation importante du nombre d'étudiants étrangers individuels à Rennes. Or les étudiants individuels sont davantage susceptibles de rencontrer des difficultés que les autres étudiants, ce qui représente un élément de préoccupation pour les acteurs publics. L'arrivée importante d'étudiants étrangers individuels et d'étudiants issus des pays du Sud a provoqué, au niveau national, des réactions politiques vives et des discours en faveur de la sélection des étudiants étrangers et d'une politique de quotas. Face aux pays anglo-saxons qui ont déjà choisi leur modèle, le traitement essentiellement commercial de l'accueil des étudiants étrangers, la France cherche encore sa voie. Afin de mieux comprendre les fluctuations quantitatives des effectifs d'étudiants étrangers, il est nécessaire de se rapporter à l'évolution des représentations et des politiques d'accueil de la France. Cela met en évidence le caractère paradoxal de la politique d'accueil actuelle caractérisée par de nombreuses contradictions : entre fermeture des frontières et essor des mobilités étudiantes, entre forte demande des étudiants issus du continent africain et sélection des étudiants étrangers en leur défaveur...

\section{Les paradoxes de la politique d'accueil des étudiants étrangers}

\section{LOGIQUES ET POLITIQUES CONTRADICTOIRES}

L'évolution des flux d'étudiants étrangers en France est directement liée à la chronologie des politiques d'accueil ainsi qu'aux logiques qui sous-tendent ces politiques. Les différents enjeux liés à l'accueil des étudiants étrangers peuvent s'accorder mais ils peuvent aussi entrer en contradiction. V. Borgogno et J. Streiff-Fénart (1996-1997) ont repéré quatre logiques à l'œuvre dans la gestion des étudiants étrangers :

- la logique universitaire qui met en évidence l'esprit universaliste et désintéressé de la transmission des connaissances. Cette vision renvoie à la mission de l'université comme ascenseur social accessible à tous. S'annexe à cette logique celle de la coopération et de la solidarité internationale dans le sens où les universités des pays développés participent à la formation des étudiants originaires des pays du Sud, notamment ceux où l'enseignement supérieur est défaillant ${ }^{11}$;

- la logique marchande qui, à l'opposé, s'inscrit dans une philosophie instrumentaliste visant à ajuster l'université aux nécessités du monde économique et à protéger les diplômes et les débouchés dans une logique de marché concurrentiel, tendance qui tend à s'affirmer dans la période actuelle. Ce point de vue conduit à considérer les étudiants étrangers comme une manne financière importante, ce qui incite certains pays comme le Royaume-Uni à faire payer aux étudiants étrangers des droits d'inscriptions plus élevés qu'aux étudiants britanniques;

- la logique de rayonnement international, selon laquelle l'accueil d'étudiants étrangers touche aux intérêts politiques et diplomatiques du territoire. De fait, l'accueil de certaines nationalités d'étudiants a longtemps été considéré comme un moyen d'assurer l'influence de la France dans le monde, en contribuant notamment au maintien de la francophonie dans les anciennes colonies, surtout dans un contexte de mondialisation qui tend à imposer l'anglais comme langue scientifique. C'est la vision prédominante dans les services culturels du Ministère des Affaires étrangères;

11. Rappelons qu'en vertu du décret n 84-431 du 6 juin 1984, les enseignants-chercheurs « contribuent au sein de la communauté scientifique et culturelle internationale à la transmission des connaissances et à la formation. Ils contribuent également au progrès de la recherche. Ils peuvent se voir confier des missions de coopération internationale ». 
- la logique du soupçon face au risque migratoire. De ce point de vue, les étudiants étrangers sont une catégorie particulière d'étrangers dont on gère les conditions d'entrée et de séjour sur le territoire d'abord en tant qu'étrangers. Cette gestion incombe au Ministère de l'Intérieur et aux préfectures (Borgogno et Steiff-Fenart, 1996-1997).

Les politiques d'accueil adoptées par les gouvernements français ont évolué au fil du temps et des circonstances en fonction des priorités accordées à telle ou telle logique. Durant la période qui suit les indépendances, l'accueil des étudiants étrangers est célébré dans les discours officiels comme un élément de la politique de prestige de la France, manifestant à la fois ses valeurs de générosité et d'ouverture et l'influence de sa culture dans le monde. L'accueil des étudiants étrangers répond alors aux intérêts de la France dans ses relations internationales d'une part, aux objectifs d'aide au développement d'autre part. Cette ouverture idéologique à l'accueil des étudiants étrangers va alors de pair avec une législation très libérale de l'entrée et du séjour des étudiants étrangers et par conséquent entraîne une forte croissance des effectifs entre 1964 et 1985.

Les années 1970 marquent le début de l'altération de cette représentation positive des étudiants originaires des pays du Sud. La crise économique amène de plus en plus à considérer tous les immigrants comme une charge pour la France. Le changement de contexte économique intervient de plus au moment où les conditions proprement universitaires de cette migration apparaissent elles aussi profondément modifiées. Entre 1960 et 1984, le nombre d'étudiants étrangers est passé de 19568 à 135000 alors même que le nombre d'étudiants nationaux a connu lui aussi une croissance considérable. Si à cette époque les nouveaux campus des années 1960 et 1970 ont permis de faire face à l'augmentation des effectifs étudiants, cela n’a plus été le cas dans les années 1980, où la croissance des effectifs s'est accélérée. La France comptait 950000 étudiants en 1985, soit cinq fois plus qu'en 1960 (200000) (Séchet et Peyon, 1993). L'insuffisance du nombre de places dans les universités est alors un argument supplémentaire en faveur de l'adoption d'une série de mesures restrictives, dans le but de freiner les demandes d'inscription des étudiants étrangers issus des pays du Sud. Pour la première fois, les étudiants étrangers sont considérés comme une catégorie particulière d'immigrés plus que comme une catégorie particulière d'étudiants. Ces étudiants sont perpétuellement soupçonnés d'être des immigrés potentiels cherchant à passer entre les mailles du filet en prenant le statut d'étudiant étranger. Les critères de délivrance des visas font partie des instructions consulaires. Plus un pays est considéré à fort risque migratoire, plus l'application des critères d'attribution des visas est restrictive. C'est pourquoi de nombreux étudiants étrangers sont soumis à des contrôles administratifs contraignants et incessants. Cette politique d'accueil a pour conséquence directe la diminution du nombre d'étudiants étrangers en France entre 1984 et 1998.

Dans la deuxième moitié des années 1990, on constate un revirement dans la position du gouvernement français. Plusieurs rapports officiels (Claeys, 1999) critiquent la position française et soulignent la diminution préoccupante du nombre d'étudiants étrangers en France du fait de cette politique migratoire restrictive. Le gouvernement Jospin décide donc d'assouplir la politique nationale concernant la délivrance des visas afin de replacer la France sur le marché mondial de la formation. La France passe alors à l'offensive par le biais d'une collaboration plus forte entre les ministères de l'Education nationale et des Affaires étrangères, une forte représentation dans les salons étrangers de l'éducation, la création de l'agence Edufrance, chargée principalement de prospecter les marchés et de promouvoir l'offre française de formation. Ces mesures ont eu pour effet une très forte croissance du nombre d'étudiants étrangers : en 2004, ils étaient 200 000, soit $60 \%$ de plus qu'en 1998. Cependant, les étudiants étrangers qui arrivent de nouveau en grand nombre sont les mêmes que ceux que l'on rejetait au moment des politiques restrictives, c'est-àdire les étudiants issus du continent africain. Étant donné que la très forte croissance du nombre d'étudiants étrangers mobiles en Bretagne est principalement due à l'arrivée importante d'étudiants africains, il est intéressant de focaliser notre attention sur ce groupe, d'autant plus que ces 
étudiants restent très peu connus des services des relations internationales des établissements ${ }^{12}$. Ces derniers viennent le plus souvent à titre individuel et sont donc difficilement identifiés par les services de la scolarité parmi la masse des étudiants français.

\section{LA QUESTION DES ÉTUDIANTS AFRICAINS}

Pourquoi, plus de cinquante années après la décolonisation, les étudiants africains continuentils d'arriver en nombre en France et quelles sont les réactions politiques face à ce nouvel afflux? Les chiffres suggèrent qu'il existe une très forte demande de la part des étudiants africains pour venir étudier dans l'enseignement supérieur français. En Bretagne, quatre étudiants étrangers expatriés sur dix sont originaires du continent africain, pour la plupart venus du Maghreb et de l'Afrique francophone. Dès que les frontières s'ouvrent, ces étudiants arrivent en grand nombre sur le territoire.

Au lendemain des indépendances, la progression des étudiants originaires des pays fraîchement décolonisés traduisait la difficulté de ces pays à répondre à la forte croissance de la demande en enseignement supérieur. Non seulement les équipements universitaires légués par l'ancien colonisateur étaient pratiquement inexistants, mais le personnel qualifié était en nombre insuffisant. Ces pays ont eu à former rapidement un grand nombre d'enseignants. Le second afflux d'étudiants africains, qui advient après l'assouplissement des règles d'entrée en France en 1998, s'explique également par l'insuffisance de l'offre universitaire au Maghreb et en Afrique francophone. Il est fréquent qu'il n'y ait pas assez de places dans les universités locales pour accueillir tous les titulaires du baccalauréat, ce qui explique le nombre important d'étudiants africains qui souhaitent intégrer l'Université française dès le premier cycle.

L'augmentation des effectifs d'étudiants en Afrique est due à la fois à l'accroissement de la population jeune et à l'amélioration significative des taux bruts de scolarisation suite à des politiques de démocratisation de l'enseignement. Notons que cette croissance des effectifs a été beaucoup plus rapide dans les pays francophones, en grande partie à cause des dispositifs d'admission en vigueur dans ces pays, calqués sur le modèle français et qui permettent à tout étudiant détenteur du baccalauréat de s'inscrire à l'Université. À titre d'exemple, il est normal de trouver dans certaines universités francophones, plus de 2000 étudiants entassés dans un amphithéâtre prévu pour 700 personnes et dépourvus des facilités requises pour assurer un enseignement de qualité (Unesco, 2003). L'enseignement supérieur en Afrique subsaharienne se caractérise également par une insuffisance des ressources financières nécessaires pour répondre de manière adéquate aux exigences d'une formation et d'une recherche de qualité. Cette situation a conduit à une détérioration sensible des infrastructures académiques et de recherche, à une réduction de la capacité d'encadrement des étudiants, à l'insuffisance des matériels d'apprentissage et, en dernière analyse, à une baisse de la qualité de l'enseignement et de la recherche. Du fait d'un manque de filières spécialisées et de la déficience de la recherche en Afrique, les étudiants issus de ce continent sont également très nombreux à souhaiter venir en France pour suivre une formation de troisième cycle, et aujourd'hui un master. De plus, les universités africaines ont connu des " années blanches ", c'est-à-dire des périodes entières sans scolarité à cause des grèves à répétition.

Cette forte pression migratoire des étudiants africains s'oppose, à différents niveaux, aux logiques qui dominent actuellement la gestion de l'accueil des étudiants étrangers en France. Depuis 2003, les critères d'attribution des visas «étudiant » et des bourses ont été précisés afin de répondre aux objectifs qualitatifs d'une politique d'accueil visant à sélectionner les étudiants étrangers en fonction des besoins et des intérêts économiques de la France. Cette volonté s'inscrit dans la nouvelle politique d'immigration initiée par le ministre de l'Intérieur d'alors, N. Sarkozy, qui

12. Les services des relations internationales des universités n’ont à leur charge administrative que les étudiants étrangers en programme d'échange ou concernés par un accord bilatéral. 
oppose « immigration choisie » et «immigration subie ${ }^{13}$ ». L'accès en master et en doctorat ainsi que l'orientation vers les disciplines scientifiques, juridiques et de gestion sont privilégiés. Des zones géographiques prioritaires sont définies : les pays scientifiquement et technologiquement développés, les pays émergents, notamment d'Asie (Chine, Inde) et d’Amérique latine (Brésil, Mexique) et les nouveaux pays de l'Union européenne.

Cette politique de sélection des étudiants étrangers a entraîné un ralentissement de l'accroissement des effectifs. L'augmentation du nombre de visas attribués est quatre fois moins importante en 2003 qu'en 1999 (7\% contre $28 \%$ ) (HCI, 2003). L'attribution des cartes de séjour mention «étudiant » confirme la tendance à la baisse. En 2003, le nombre de titres de séjour attribués a diminué de 4800 par rapport à 2002. L'Afrique n'étant pas comme la Chine un pays émergent à fort potentiel de développement économique avec lequel chaque pays souhaite développer des liens commerciaux étroits, elle ne fait pas partie des zones géographiques privilégiées. Entre 1995 et 2003, près de 700 programmes internationaux ont été créés entre des universités chinoises et des universités étrangères (Raizon, 2005) : «La France et les pays anglo-saxons se disputent ce «marché » que représentent les étudiants chinois, dont le nombre ne cesse d'augmenter» (Sztanke, 2005). La forte demande chinoise pour l'enseignement supérieur des pays développés est perçue davantage comme une opportunité de créer des liens et des débouchés économiques que comme un «souci migratoire »., contrairement à la demande africaine.

La politique de l'immigration choisie réaffirme le fait de privilégier l'arrivée des étudiants étrangers dans le cadre de programmes d'échanges internationaux. Depuis le début des années 1980, la coopération Nord-Sud, soutenue par une éthique de solidarité, cède la place à une nouvelle conception des échanges universitaires internationaux, qui les inscrit dans une logique de marché de la formation et de l'emploi hautement qualifié. Cette nouvelle conception de la coopération met en avant l'équivalence des valeurs, la réciprocité des échanges et la compétitivité et s'inscrit pleinement dans la création, en 1987, du programme d'échange européen Erasmus à l'origine de l'européanisation des effectifs des étudiants étrangers en France. Encore une fois, l'Afrique, de par la défaillance de son enseignement supérieur, ne peut répondre à ce critère de "réciprocité des avantages », et, de fait, se retrouve exclue des nouvelles dispositions concernant les échanges universitaires. Par conséquent, ces étudiants africains arrivent en grande majorité à titre individuel, sans encadrement, ni aide financière. De plus, le nombre de boursiers du gouvernement français a diminué de $21 \%$ entre 2001 et 2003, passant de 24360 à 19171 (Szymankiewicz, 2005). Aussi, beaucoup viennent sans bourse en France, comptant sur d'autres ressources, l'aide d'un parent ou d'un proche, un travail à temps partiel, la prise en charge par la communauté des compatriotes... Cependant, cette situation peut vite devenir problématique si les revenus escomptés ne se concrétisent pas.

Dans la continuité des politiques restrictives mises en place dans les années 1970, la délivrance du visa et du titre de séjour est subordonnée à la présentation de ressources d'un montant au moins égal au montant des bourses accordées par le gouvernement français (environ 450 euros/mois). Les préfectures demandent de façon quasi systématique les relevés de compte des étudiants étrangers. Pour bon nombre d'étudiants africains non-boursiers, qui se retrouvent dans une détresse financière, particulièrement depuis la dévaluation du franc CFA, il est difficile de justifier ce niveau de ressources.

De nombreux étudiants africains sont confrontés à des situations de grande précarité et sont dans l'obligation de faire appel à l'aide sociale du CROUS : "Près de $40 \%$ des bénéficiaires du Fonds de Solidarité Universitaire, le principal fonds d'aide d'urgence destiné aux étudiants, sont des étudiants étrangers, dont $85 \%$ "d'individuels" ( Szymankiewicz, 2005). Un rapport de la Direction Régionale de l'Équipement de la Bretagne sur L'évolution des besoins en logement des étudiants montre l'existence de nombreuses situations de précarité : « La situation des étudiants

13. Loi du 26 novembre 2003 relative à la maîtrise de l'immigration, au séjour des étrangers en France et à la nationalité. Loi du 24 juillet 2006 relative à l'immigration et à l'intégration 
étrangers qui sont recrutés hors convention à titre individuel est clairement problématique : sur 522 en 2003, 400 ont été identifiés comme étant en situation de précarité. Or l'augmentation des étudiants étrangers accueillis dans le cadre de conventions, logés prioritairement dans le parc du CROUS, exclut de ce fait une fraction importante des étudiants non boursiers à la limite des plafonds et les étudiants étrangers "individuels" précarisés » (DRE, 2004). Cet exemple du logement montre bien que les acteurs locaux, région, villes ou universités sont en première ligne dans la gestion des effets des politiques nationales. La question est particulièrement sensible en Bretagne, où la tradition d'accueil n'est pas ancienne et où les étudiants venant à titre individuel sont de plus en plus nombreux.

\section{Conclusion}

La Bretagne connaît, depuis quelques années, une des plus fortes croissances du nombre d'étudiants étrangers expatriés en France. Ce «boom » de la présence étrangère dans l'enseignement supérieur qui soulève de nombreuses questions, que cela soit en termes d'adaptation des structures d'accueil ou de stratégies politiques d'ouverture internationale de la région. Or, même si l'échelon régional dispose d'une marge d'action de plus en plus importante, les décideurs nationaux restent aux commandes des premiers leviers de l'accueil des étudiants étrangers, à travers la politique d'immigration. La gestion de l'accueil des étudiants étrangers est donc difficile, compte tenu de l’implication de différentes échelles de décisions et des logiques contradictoires qui sous-tendent les politiques d'accueil. D'après S. Slama (1999), il y a toujours une tendance qui prédomine et qui «écrase » les autres; cet auteur parle de «l'impossible politique de l'étudiant étranger » et affirme que la logique des flux migratoires parvient toujours à s’imposer aux autres.

Le traitement de la catégorie d'étudiant étranger dans les statistiques officielles vient confirmer cette affirmation. Statistiques et politiques font continuellement l'amalgame entre mobilité étudiante et immigration de travail. La sélection des étudiants étrangers a été proposée comme une solution à la contradiction entre politique migratoire et rayonnement international de l'Université française. Cependant, elle ne vient pas résoudre la question de la forte demande des étudiants des pays du Sud pour l'enseignement supérieur français et, au contraire, aurait plutôt tendance à participer au cercle vicieux de la fuite des compétences dont sont victimes ces pays en voie de développement. En effet, en privilégiant les étudiants étrangers des $2^{\mathrm{e}}$ et $3^{\mathrm{e}}$ cycles $^{14}$, cette politique d'accueil renforce la pénurie de jeunes chercheurs dans les pays du Sud, cause principale du manque d'enseignants-chercheurs et de formations, ce qui contribue au départ des étudiants vers des universités étrangères. Comment peut-on alors concilier accueil des étudiants étrangers, lutte contre la fuite des compétences et solidarité internationale?

\section{Bibliographie}

Aubert (F.), Tripier (M.), Vourc'H (F.), 1996. - « Trajectoires sociales, origines nationales et modes de vie étudiants. Comparaison entre étudiants "français", "étrangers", "issus de l'immigration” », Migrantsformation, $\mathrm{n}^{\circ} 104$, p. 111-129.

Borgogno (V.), Steiff-Fenart (J.), 1996-1997. - « L'accueil des étudiants étrangers en France : évolution des politiques et des représentations », Les cahiers du Soliis, Notes et Travaux Sociologiques, n 2-3, p. $73-88$.

Borgogno (V.), Vollenweider (L.), 1998. - «Les étudiants étrangers en France : trajectoires et devenir, nouveaux éclairages », Migrations Études, n 79, Université de Nice-Sophia Antipolis, 12 p.

14. La nouvelle politique d'immigration votée en 2006 donne la possibilité aux étudiants étrangers diplômés d'un master ou d'un doctorat de rester 6 mois après leurs études pour trouver un travail en France. 
Claeys (A.), 1999. - L'accueil des étudiants étrangers en France : enjeu commercial ou priorité éducative?, Rapport d'information n 1806, Paris, Assemblée Nationale, 130 p.

Coulon (A.), Paivandi (S.), 2003. - Les étudiants étrangers en France : l'état des savoirs, Rapport pour l'Observatoire de la Vie Étudiante, Université de Paris 8, 53 p.

DEP, 2005. - Repères et références statistiques, Paris, Ministère de l'éducation nationale, 383 p.

DRE Bretagne, 2004. - L'évolution des besoins en logement des étudiants, Rapport de la division de l'aménagement et de l'habitat, Rennes, $40 \mathrm{p}$.

FABRE (J.), Guillerm (M.), 2007. - Les étudiants étrangers dans l'enseignement supérieur français : une croissance soutenue par les Asiatiques, Note d'Information 07.02, DEP, Ministère de l'Éducation Nationale, Paris, 6 p.

HCI (Haut conseil à l'Intégration), 2003. - Observatoire statistique de l'immigration et de l'intégration, Rapport 2002/2003, HCI, Paris, 63 p.

Jallade (J.-P.), Gordon (J.), Lebeau (N.), 1996. - La mobilité étudiante au sein de l'Union Européenne : une analyse statistique, Rapport publié par la commission européenne, European Institute of Education and Social Policy, Bruxelles, 80 p.

LAFFORT (B.), 2005. - « Le mythe du retour », Hommes et Migrations, n 1253, p. 105-116.

ONU, 2004. - Annuaire statistique, 48 édition (données disponibles au 15 décembre 2003), Nations Unis, New-York, 894 p.

OCDE, 2003. - Regards sur l'éducation, les indicateurs de l'OCDE, Paris, p. 302-303.

RaIzon (D.), 2005. - «Comment sont sélectionnés les étudiants étrangers? », Radio France International, article disponible sur le site Web de RFI [http://www.rfi.fr/actufr/articles/067/article_37372.asp].

Renaudat (C.), 1998. - Les étudiants africains à Bordeaux, Centre d'étude d'Afrique Noire, Études et Prospectives, UMR 206 CNRS, Talence, 112 p.

SÉChet (R.), Peyon (J.-P.), 1993. - Les Universités de Nantes et de Rennes et leurs antennes : espace imaginé, espace approprié, espace promotionnel, Rapport final, Appel d'offres de recherche «l'Université et la ville ", PUCA, Paris, 181 p.

Slama (S.), 1999. - La fin de l'étudiant étranger, Paris, L'Harmattan, 312 p.

SzTANKe (M.), 2005. - « Pékin - Paris : l'étudiant chinois est-il une marchandise? », Hommes et Migrations, $\mathrm{n}^{\circ} 1254$, p. $74-81$.

SzYMANKIEWICZ (C.), 2005. - Les conditions d'inscription et d'accueil des étudiants étrangers dans les universités, Rapport du ministère de l'Éducation, Paris, 105 p.

TABET (M.-C.), 2005. - «Étudiants étrangers, le grand trucage », Le Figaro, 28 septembre, p. 9.

TERRIER (E.), 2005. - Les étudiants étrangers en Bretagne : analyse démographique et présentation des enjeux, Mémoire de Master 2 Recherche, Université Rennes 2, 194 p.

Teissier (C.), Theulière (M.), Tomasini (M.), 2004. - Les étudiants étrangers en France, ministère de l'Éducation Nationale, de l'Enseignement supérieur et de la Recherche, Les dossiers n 153, DEP, Paris, $55 \mathrm{p}$.

UNESCO, 2003. - Développements récents et perspectives de l'enseignement supérieur en Afrique subsaharienne au XXI siècle, document préparé par les bureaux de l'Unesco à Dakar et à Harare, Paris, UNESCO, $33 \mathrm{p}$.

Vollenweider-Andersen (L.), 1996-1997. - « Les étudiants étrangers dans l'enseignement supérieur français : données statistiques », Les cahiers du Soliis, Notes et Travaux Sociologiques, n² 2-3, p. 61-72.

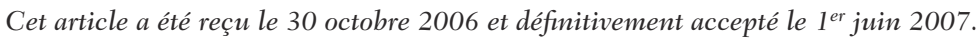

